\title{
Understanding Development and Lateralization of Major Cerebral Fiber Bundles in Pediatric Population Through Quantitative Diffusion Tensor Tractography
}

\author{
RICHA TRIVEDI, SHRUTI AGARWAL, RAM KISHORE S. RATHORE, SONA SAKSENA, RAJENDRA P. TRIPATHI, \\ GYANENDRA K. MALIK, CHANDRA M. PANDEY, AND RAKESH K. GUPTA
}

\begin{abstract}
Departments of Radiodiagnosis [R.T., S.S., R.K.G.] and Biostatistics [C.M.P.], Sanjay Gandhi Postgraduate Institute of Medical Sciences, Lucknow 226014, India; Department of Mathematics and Statistics [S.A., R.K.S.R.], Indian Institute of Technology, Kanpur 208016, India; Department of Radiodiagnosis [R.P.T.], Institute of Nuclear Medicine and Allied Sciences, New Delhi 110054, India; Department of Pediatrics [G.K.M.], Chhatrapati Shahuji Maharaj Medical University, Lucknow 226018, India
\end{abstract}

\begin{abstract}
Region of interest based morphometric diffusion tensor imaging analysis, has been used extensively for the assessment of age-related changes in human brain, is limited to two dimensions and does not reflect the whole fiber bundle; however, diffusion tensor tractography (DTT) offers an overall view of individual fiber bundle in three-dimensional spaces. Quantitative DTT was performed on 51 healthy subjects of pediatric age range and young adults to compare age-related fractional anisotropy (FA) changes in corpus callosum, sensory and motor pathways, limbic tracts [cingulum (CNG) and fornix $(\mathrm{Fx})]$, and superior and inferior longitudinal fascicules. In corpus callosum, inferior longitudinal fascicules, limbic tracts $(\mathrm{CNG}$ and Fx), sensory pathways, and motor pathways, an initial sharp increase in FA was observed up to the age of $2 \mathrm{y}$ followed by a gradual increase up to $21 \mathrm{y}$. In superior longitudinal fascicules, sharp increase in FA was observed up to 3 y followed by a gradual increase. The FA value of the left CNG ( $p=0.01$, sign test) was observed to be significantly greater than that of the right CNG. We conclude that white matter fiber tracts mature with age and can be assessed by using DTT that may greatly improve our understanding of the human brain development. (Pediatr Res 66: 636-641, 2009)
\end{abstract}

$\mathrm{I}^{\mathrm{n}}$ humans, a great deal of the axonal organization is established by birth; however, axonal wiring and pruning processes as well as myelination in white matter (WM) tracts are known to extend to the postnatal periods and continues till adulthood (1-3). Postmortem studies of the young population are very limited (4). Even with postmortem samples, there are no reliable histology-based techniques that can quantitatively assess the axonal architecture of the entire fiber tracts.

Conventional magnetic resonance imaging (MRI) has provided an opportunity to visualize the progression of myelination in WM tracts throughout infancy and childhood noninvasively (5). Magnetization transfer imaging has been shown to be sensitive for the evaluation of brain maturation (6). However, conventional MRI is unable to delineate individual WM fiber tracts. Recent developments in diffusion MRI have put this imaging modality to forefront of neuroscience re-

Received February 9, 2009; accepted August 4, 2009.

Correspondence: Rakesh K. Gupta, M.D., Department of Radiodiagnosis, Sanjay Gandhi Post Graduate Institute of Medical Sciences, Raebareli Road, Lucknow, UP 226014, India; e-mail: rgupta@sgpgi.ac.in or rakeshree1@gmail.com

Supported by grant no. BT/PR5009/MED/14/581/2004 from the Department of Biotechnology, New Delhi, India. search. By virtue of its diffusion-driven displacement, water molecules probe tissue structure at a microscopic scale in diffusion imaging (7). It has been shown that ordered axonal structure, cell membrane, and myelin sheath strongly influence water diffusion (8) that directly links it to axonal orientation and integrity in the brain (9). Diffusion tensor imaging (DTI) allows us to estimate dominant fiber orientation at each pixel. The most commonly used DTI analysis is based on a two dimensional (2D) region of interest (ROI) in an image dataset that is user specific. DTI studies in healthy pediatric and adult individuals using 2D ROI approach indicate that mean diffusivity (MD) in central WM tracts decreases with age, whereas fractional anisotropy (FA) increases up to the age of $70 \mathrm{y}$. After $70 \mathrm{y}$, increasing trend of MD along with decreasing trend of FA has been shown in central WM tracts (10). This method is not always reliable because of the uncertainty of manual specification. In addition, ROI-based morphometric DTI analysis is limited to 2D and does not reflect the whole fiber bundle; however, diffusion tensor tractography (DTT) offers an overall view of individual fiber bundle in $3 \mathrm{D}$ spaces (11). In addition to basic 3D visualization, many studies have used fiber tracking to delineate specific WM tracts for quantitative analysis. Quantitative DTT studies have examined the microstructure of WM tracts in the various (subset) age groups of pediatric subjects (12-16), in elderly subjects $(13,17)$, and in patients with various neuropathologies $(18,19)$. However, there is no DTT study covering entire pediatric range from neonates (within 1 mo of age) to adolescent (18 y of age). To the best of our knowledge, this is the first cross-sectional quantitative DTT study in the entire range of pediatric population. The aim of our study was to compare temporal changes in FA values of major WM fiber tracts in 3D space and to look for cerebral laterality.

\footnotetext{
Abbreviation: CC, corpus callosum; CNG, cingulum; DTI, diffusion tensor imaging; DTT, diffusion tensor tractography; D, dimensions; FA, fractional anisotropy; FD, fiber density; Fx, fornix; ILF, inferior longitudinal fascicules; MD, mean diffusivity; ROI, region of interest; SFM, stable fiber mass; SLF, superior longitudinal fasciculus; WM, white matter.
} 


\section{MATERIALS AND METHODS}

Conventional MRI and DTI were performed on normal human brain $[n=51$, nine neonates (with in $1 \mathrm{mo}$ ), nine infants (1 mo-1 y), 24 children (1-12 y), six adolescent (13-18 y), and three young adults $(>18 \mathrm{y})$ ] with age group ranging from $10 \mathrm{~d}$ to $21 \mathrm{y}$. The subjects consisted of 21 males and 30 females (median age $=12 \mathrm{y}$ ). Handedness in children and adolescent was assessed using a standardized questionnaire (Edinburgh inventory) (20). All the children and adolescent included in this study were right handed. However, in neonatal and infant age group handedness was assessed by grasp reflex analysis (21). Left-handed neonates as well as infants were excluded from study. Twelve of 18 neonates and infants were right handed and remaining six subjects were mixed handers. Healthy volunteers were recruited from the community who had come for routine brain screening with no neurologic symptoms. Small healthy babies formed the controls in project relating to hypoxic ischemic encephalopathy. These controls were taken with institutional ethical approval. Parents of these neonates and children were anxious and wanted to get MRI to confirm that their children brain had no apparent imaging abnormalities. Rest of the subjects included in this study came to us with complain of isolated mild peripheral joint injury, and they were neurologically normal on imaging and performed well on serial neurologic examinations. In all subjects, MRI scans were performed without any sedation. Written consent for performing these studies was obtained from the parents.

Imaging parameters. Imaging was performed on a 1.5 Tesla GE MRI scanner (GE Healthcare Technologies, Milwaukee, WI) using a standard birdcage quadrature head coil. MR imaging parameters included: T2weighted axial fast spin-echo (SE) images [repetition time (TR) $=6000 \mathrm{~ms}$, echo time $(\mathrm{TE})=85 \mathrm{~ms}$, and number of excitation $(\mathrm{NEX})=4$ ]; T1-weighted axial SE images $(\mathrm{TR}=700 \mathrm{~ms}$, $\mathrm{TE}=14 \mathrm{~ms}$, and $\mathrm{NEX}=2)$; and T2-weighted fluid-attenuated inversion recovery images $(\mathrm{TR}=9000 \mathrm{~ms}$, $\mathrm{TE}=120 \mathrm{~ms}$, inversion time $=2200 \mathrm{~ms}$, and NEX $=1$ ). A total of 30 to 36 contiguous 3 -mm thick axial sections were acquired with a $240 \times 240 \mathrm{~mm}^{2}$ field of view and image matrix of $256 \times 256$.

DTI data were acquired using a single-shot echo-planar dual SE sequence with ramp sampling. The diffusion-weighted acquisition parameters were: b-factor $=0,700$ (in subjects of age $10 \mathrm{~d}-12 \mathrm{mo}$ ), $1000 \mathrm{~s} / \mathrm{mm}^{2}$ (in rest of the subjects), slice thickness $=3 \mathrm{~mm}$ with no gap, field of view $=240 \times 240$ $\mathrm{mm}^{2}, \mathrm{TR}=8 \mathrm{~s}, \mathrm{TE}=100 \mathrm{~ms}$, and NEX $=8$. A total of 30 to 36 axial sections were acquired with an image matrix of $256 \times 256$ (following zero-filling). The diffusion tensor encoding used was the balanced rotational invariant with 10 noncollinear directions over the unit hemisphere (22). Imaging was done carefully in all subjects. There was evidence of motion in six studies and were excluded from the study. Segmentation of WM structures and DTT was performed using in-house developed JAVA based software (abstract no. 3540 published in proceedings of ISMRM 2009) described below.

Segmentation of WM structures. The key idea of our method (Fig. 1) is to do a segmentation of the principal eigenvector field into stable voxels having a minimal e1 variation (curvature). Thus, a voxel $P$ is a member of the

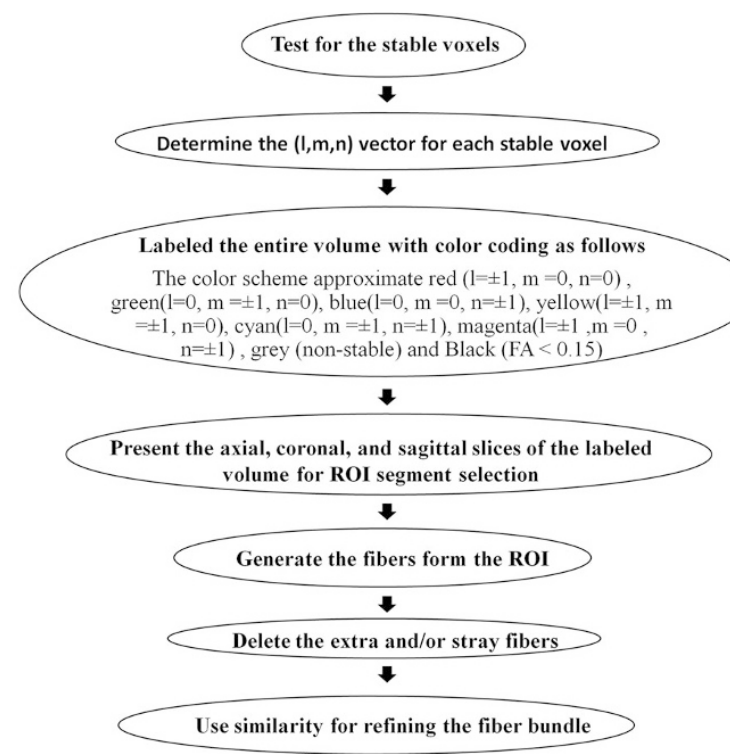

Figure 1. Flow chart explaining the algorithm used for fiber tracking. stable fiber mass, if there is a neighboring voxel $Q$ such that e1's at $P$ and $Q$ point out to each other. Mathematically, it translates to the relation $G(F(P))=$ $P$, where with $u=(1,1,1)$

$$
\begin{gathered}
\mathrm{F}(\mathrm{P})=\operatorname{ROUND}\left(\mathrm{P}+\mathrm{e}^{1}(\mathrm{P})+0.5 u\right) \\
\mathrm{G}(\mathrm{Q})=\operatorname{ROUND}\left(\mathrm{Q}-\mathrm{e}^{1}(\mathrm{Q})+0.5 u\right)
\end{gathered}
$$

The method, thus, firstly generates the stable fiber mass and then segments it by coloring its voxels according to the following scheme. The vector joining $P$ and $Q$ has form $(l, m, n),(l, m, n=-1,0,1)$ using which the voxel $P$ is colored according to table. Typical segmented axial, sagittal, and coronal color maps were generated. Using these color maps, our method narrows down the ROIs selection to pointing out to a color segment inside a broader ROI through a single mouse click.

Diffusion tensor tractography. Different fiber bundles are easily reconstructed by the segmented components using fiber assignment by continuous tracking algorithm (23). This reconstruction allowed us to identify the coordinates of specific WM tracts and to investigate the anatomy. DTI measures were calculated for the entire fiber and reported. FA threshold of 0.15 was used for fiber tracking.

Fiber density. Consider a r-cube centered at voxel $P$. FD at point $P$ (with respect to length $r$ ) is defined to be the number of voxels in this cube belonging to the fiber mass divided by the volume of the voxel $\left(r^{3}\right)$. This is the relative volume occupied by voxels lying on fibers. Computations taking $r=$ 7 seems to be suitable. Reported results were taken for $r=7$.

DTI indices derived from the entire fiber bundle were averaged for the purpose of quantitation.

Data quantification. The mouse clicks were made on midsagittal stable fiber mass map on CC at the level of massa intermedia (Fig. 2A).

The mouse clicks were made on right and left fornix (Fx) on those coronal stable fiber mass map where the thickness of respective fiber bundle was maximum (Fig. $2 A$ ). In case of posterior cingulum (CNG) the mouse clicks were made on those coronal stable fiber mass map where the thickness of fiber bundle was maximum; however, for the fiber tracts generation of anterior $\mathrm{CNG}$, the mouse clicks were made on those axial stable fiber mass map where the thickness of fiber bundle was maximum.

The mouse clicks were made on right and left superior (SLF) and inferior longitudinal fasciculus (ILF) on those coronal stable fiber mass maps where the thickness of respective fiber bundle was maximum. For the minimization of interobserver variability, two investigators evaluated the entire data sets.

The central sulcus was identified and marked on sagittal surface image reconstructed by $3 \mathrm{D}$ surface rendering of $b_{0}$ image stack. Free hand ROIs were drawn on axial T2-weighted image near the brain's vertex on the precentral and postcentral gyri, and the fibers generated from those ROIs were defined as motor or sensory tracts, respectively (Fig. 2B).

Statistical analysis. DTI measures were quantified for each fiber tracts from the control brains in the age range of $10 \mathrm{~d}$ to $21 \mathrm{y}$. To explore the changes of DTI measures with respect to age, each fiber tract was quantified for various age groups at an interval of $1 \mathrm{y}$ (up to $5 \mathrm{y}$ of age) and subsequently at an interval of $5 \mathrm{y}$. One-way analysis of variance was performed to see the difference in DTI measures among all age groups. Student-Newmen-Keuls post hoc test was applied to identify the age interval responsible for the difference in these parameters.

Bivariate analysis of correlation was performed to rate inter-rater reliability between two observers, with the assumption that there was no correlation between DTI measures quantitated by two observers $\left(\mathrm{H}_{\mathrm{o}}\right.$ [null hypothesis] $=$ $0)$. Alternatively, if a correlation more than 0.001 was observed at $\alpha=0.05 \%$ and $90 \%$ power of test, the null hypothesis was rejected. To study the relationship between age and DTI measures of the fiber bundles, linear, quadratic, cubic, log-linear, growth, and exponential models were applied. For each model, regression coefficients, $R^{2}, 95 \%$ confidence interval of mean, and other model statistics were computed. $R^{2}$ statistics was used to determine the best fit model. Finally, the log-linear model was considered to examine the trends between FA values in fiber bundles with increasing age (Fig. 3). The following equation was used to estimate FA as a function of age.

$$
\mathrm{FA}=a_{1} \operatorname{Ln}(\text { age })+a_{0}
$$

where $a_{0}$ is constant term, and $a_{1}$ is regression coefficient of log transform of age.

The Sign test was used to compare the left-right asymmetry in the FA [FA(right fiber bundle) - FA(left fiber bundle)] of motor and sensory tracts, limbic tracts (CNG and Fx), SLF, and ILF and its temporal dependence. $p$ value less than 0.05 was considered to be significant. The computations were performed using the statistical package for social sciences (SPSS, V15, Inc, Chicago, IL). 


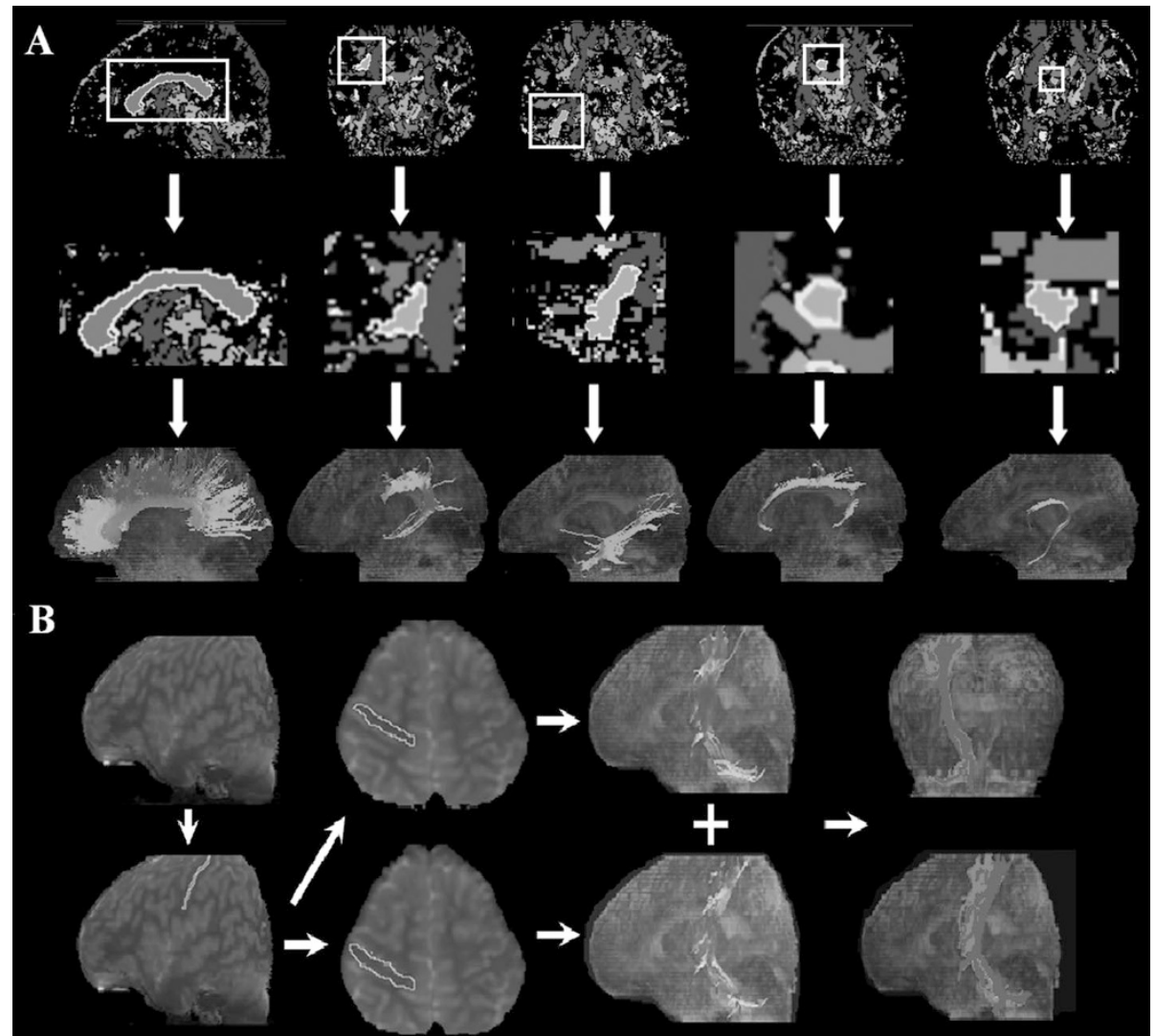

Figure 2. Demonstration of methodology used for tract reconstruction from 7-y old healthy subject. (A) Sagittal CC and coronal SLF, ILF, $\mathrm{CNG}$, and Fx stable fiber mass maps (first row) showing selection of planes for the reconstruction of respective right tract. $(B)$ Demonstration of methodology used for motor (first row) and sensory tract (second row) reconstruction.

\section{RESULTS}

The inter-rater reliability for the DTT-based approach was $0.90(p<0.01)$. Based on regression and model diagnostics, log-linear model was considered to examine the trends in FA in all fiber bundles with increasing age in the normal human brain (Fig. 3A-E, Table 1). An initial sharp increasing trend in FA followed by a gradual continuous rise was observed as a function of age in all WM fiber bundles. The lowest FA and FD values along with highest MD values in whole WM fiber bundles were observed in newborns; however, an opposite trend of highest FA and FD along with lowest MD values in these WM fiber bundles was observed in young adults.

Figure 4 shows the 3D projection of different fiber bundles on midsagittal plane at different ages.

Age-related changes in FA in $3 D$ WM fiber bundle. FA was highest in $\mathrm{CC}$ followed by ILF, motor tracts, and sensory tracts $\approx \mathrm{CNG} \approx \mathrm{Fx} \approx \mathrm{SLF}$ (Fig. 3).

Based on Student-Newmen-Keuls post hoc test, the highest increase in FA in the $\mathrm{CC}$, limbic tracts, sensory, and motor tracts was observed up to the age of $2 \mathrm{y}$ followed by a gradual continuous rise subsequently. The FA showed an initial sharp increase in SLF and ILF up to the age of 3 y followed by a gradual continuous rise subsequently.

Cerebral asymmetry-related difference. As can be seen from Fig. 3, the FA values were observed to be higher in the left fiber bundles of each tract except for Fx compared with the right fiber bundles at all ages. The FA value of the left CNG ( $p=0.01$, sign test) was observed to be significantly greater than that of the right $\mathrm{CNG}$.
Age-related changes in FD and MD in $3 D$ WM fiber bundle. An increasing trend of FD along with decreasing trend of MD values was observed as a function of age in all fiber bundles of interest in 3D space; however, no regression model provided a significant $R^{2}$ values to determine the changes in FD and MD values with age.

On comparing fiber bundle of right hemisphere and their contralateral fiber bundle, we observed no consistent pattern in FD and MD values associated with cerebral laterality for the major WM fiber bundles under study.

\section{DISCUSSION}

This study demonstrates the potential of DTT in assessment of 3D changes in major WM tracts (CC, SLF, ILF, motor and sensory tracts, and limbic tracts) with the progression of age. In this study, the pattern of log-linear graphs showed that SLF and ILF fiber bundle lags behind CC, limbic, and sensory and motor tracts. In addition, we also observed significantly higher FA values in left cerebral hemisphere compared with right in CNG at all ages suggestive of lateralization.

Previous ROI-based DTI studies in children and adults have shown that during childhood there is a fast early rise in anisotropy in the WM followed by a slower growth subsequently, and this is believed to be associated with the process of myelination $(5,24,25)$. All these studies have described age-related changes in DTI indices only in a small region of cerebral WM tracts. Manually placed ROI analysis has been widely used in the DTI literature but has intraobserver and interobserver variability and is time consuming. In addition, 

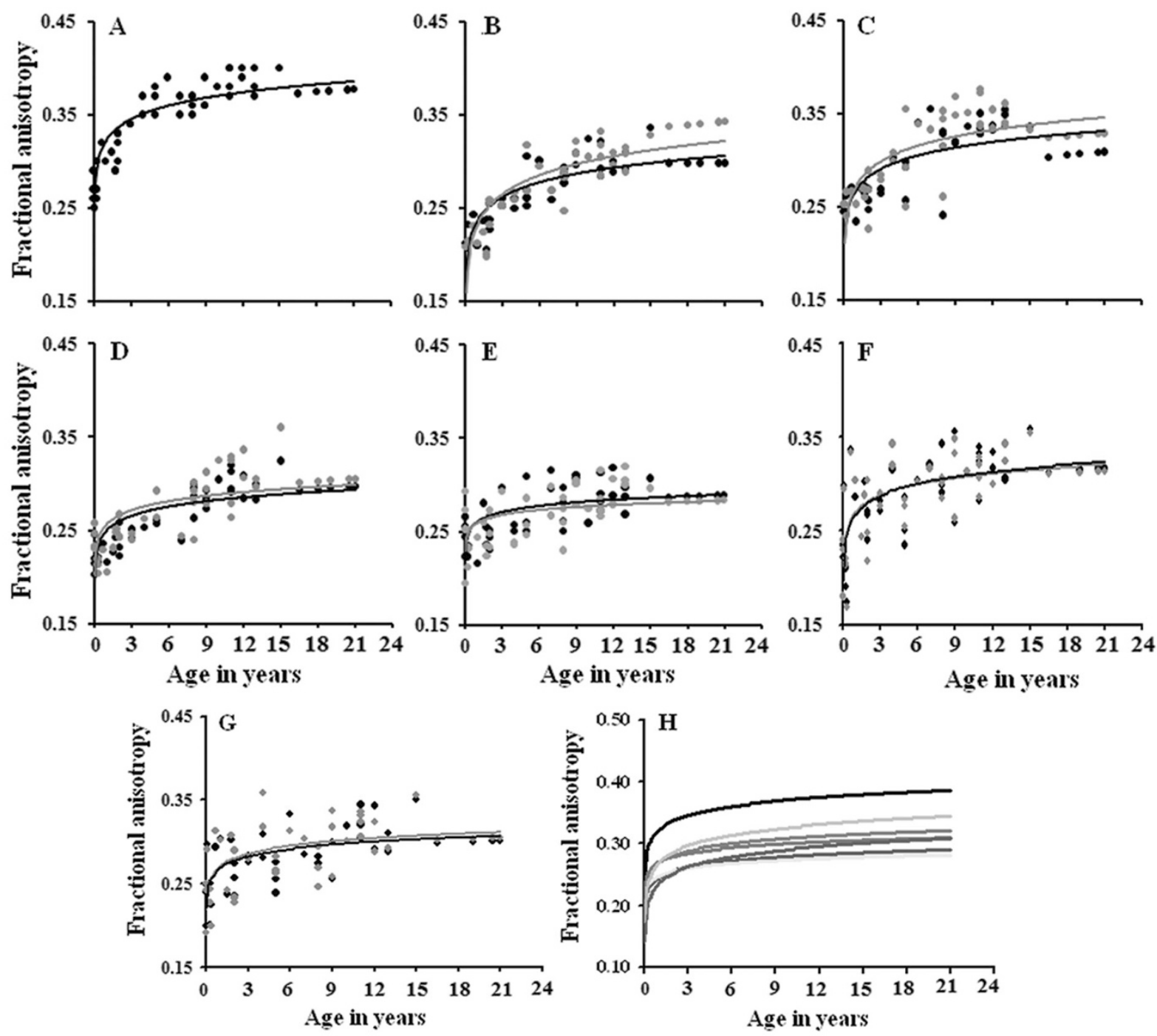

Figure 3. Relationship between FA in whole fiber bundle ( $A, \mathrm{CC} ; B$, SLF; $C$, ILF; $D, \mathrm{CNG} E$, Fx; $F$, motor pathways; and $G$, sensory pathways) and age using $\log$ linear regression model. $H$, Comparison between FA and age among different fiber bundles using log linear fit at $95 \%$ confidence limit. (Note:

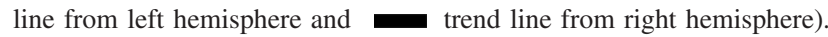

only a part of each tract can be assessed. The advantage of voxel-based analysis techniques is being observer independent and can be applied to study the entire brain. Voxel-based analysis techniques have been applied to DTI (26), and researchers are also trying to apply tract-based morphomentry analysis to DTT (27). However, morphometric analysis also

Table 1. Relationship between age and FA in white matter fiber tracts of the developing brain using log-linear regression model

\begin{tabular}{|c|c|c|c|c|c|c|}
\hline \multirow[b]{2}{*}{ Regions } & \multirow[b]{2}{*}{ FA $($ Mean \pm SD $)$} & \multicolumn{2}{|c|}{$\begin{array}{l}\text { Regression } \\
\text { coefficients }\end{array}$} & \multicolumn{3}{|c|}{ Model diagnostics } \\
\hline & & $a_{0}$ & $a_{1}$ & $R^{2}$ & $F$ & Significance \\
\hline $\mathrm{CC}$ & $0.34 \pm 0.04$ & 0.32 & 0.02 & 0.61 & 78.05 & $<0.001$ \\
\hline Right SLF & $0.27 \pm 0.03$ & 0.24 & 0.02 & 0.61 & 62.46 & $<0.001$ \\
\hline Left SLF & $0.27 \pm 0.04$ & 0.24 & 0.02 & 0.68 & 83.13 & $<0.001$ \\
\hline Right ILF & $0.30 \pm 0.04$ & 0.27 & 0.02 & 0.50 & 39.31 & $<0.001$ \\
\hline Left ILF & $0.31 \pm 0.04$ & 0.28 & 0.02 & 0.49 & 38.83 & $<0.001$ \\
\hline Right CNG & $0.26 \pm 0.03$ & 0.25 & 0.02 & 0.62 & 66.35 & $<0.001$ \\
\hline Left CNG & $0.27 \pm 0.04$ & 0.26 & 0.02 & 0.48 & 38.23 & $<0.001$ \\
\hline Right Fx & $0.27 \pm 0.03$ & 0.26 & 0.01 & 0.44 & 26.12 & $<0.001$ \\
\hline Left Fx & $0.27 \pm 0.03$ & 0.26 & 0.01 & 0.37 & 44.21 & $<0.001$ \\
\hline Right motor tracts & $0.29 \pm 0.05$ & 0.22 & 0.02 & 0.50 & 41.14 & $<0.001$ \\
\hline Left motor tracts & $0.29 \pm 0.04$ & 0.23 & 0.02 & 0.47 & 36.93 & $<0.001$ \\
\hline Right sensory tracts & $0.28 \pm 0.04$ & 0.24 & 0.01 & 0.41 & 28.43 & $<0.001$ \\
\hline Left sensory tracts & $0.29 \pm 0.04$ & 0.24 & 0.01 & 0.38 & 24.86 & $<0.001$ \\
\hline
\end{tabular}

CC, corpus callosum; SLF, superior longitudinal fasciculus; ILF, inferior longitudinal fasciculus; CNG, cingulum; Fx, fornix; FA, fractional anisotropy. 


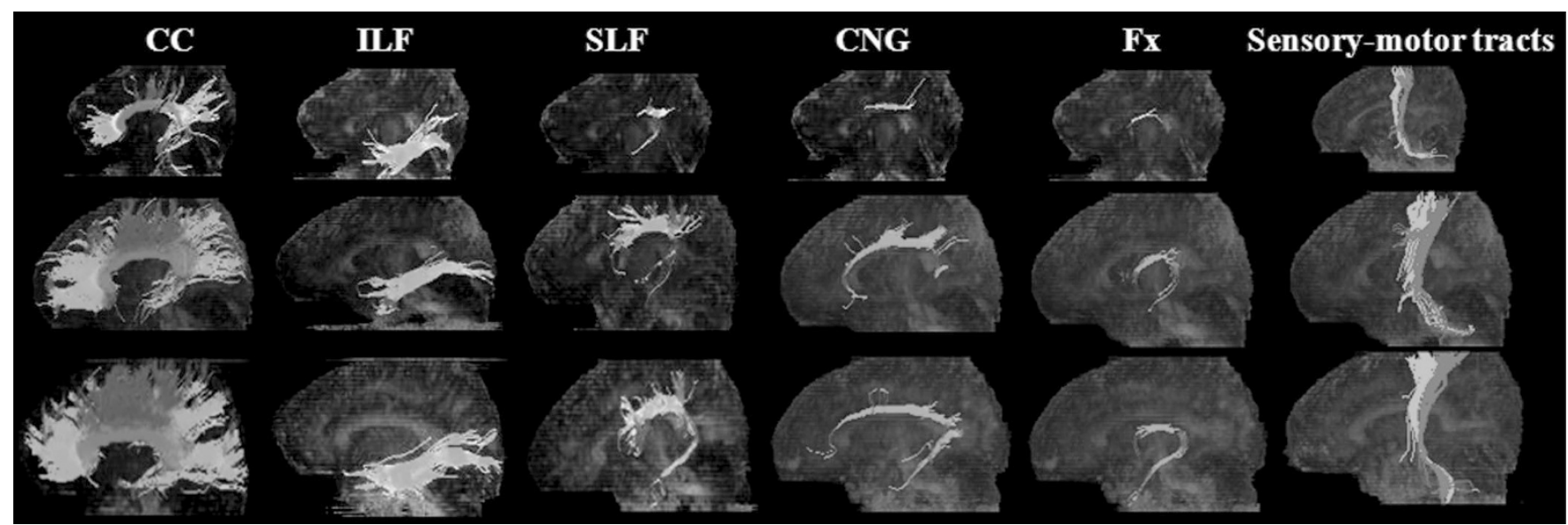

Figure 4. Projection of different fiber bundles of left hemisphere on midsagittal plane at different age: (first row) 1 mo, (second row) 4 y, (third row) 14 y shows normal pattern of fiber bundles in human brain.

has some inherent shortcomings because of the requirements of spatial normalization and smoothing.

Three-dimensional DTI fiber tracking can be used as the basis for quantitative assessment of the microstructure of a specific WM tract. Diffusion metric maps including FA, MD, and the eigenvalues are inherently registered to the resultant DTI fiber tracks. The general strategy of quantitative DTI fiber tracking is to create a 3D ROI based on the voxels through which the fiber tracks pass. Quantitative DTI fiber tracking can be performed in conjunction with deterministic or probabilistic fiber tracking. Studies have shown the reproducibility of quantitative DTI fiber tracking (28) as well as the accuracy of the technique and improved intraobserver reliability compared with manually drawn ROI. Discrepancies between the scales of the axonal diameter and the imaging voxel size (i.e. low spatial resolution), the noise contained in the diffusion data, and image artifacts comprise most of the limitations associated with tractography. DTT assumes that the fibers visualized in each voxel are well described by a single orientation estimate; therefore, the technique performs poorly in regions where fibers cross, merge, or diverge. These limitations might lead to the tracking of pathways that do not exist or the ineffective tracking of those that do exist.

The increase in FA in WM tracts with age is believed to be associated with axonal reorganization as well as ongoing process of myelination. Although in some WM tracts myelination process is documented until the third decade of life or later, majority of myelination occurs within the first $2 \mathrm{y}$ of life (1). Myelination does not occur synchronously in all WM tracts, it initiates and completes differentially among the WM fiber bundles based on the functional capacity of neurons. In our study, a sharp increase in FA was observed at the age of $2 \mathrm{y}$ in $\mathrm{CC}$, sensory and motor tracts, and limbic tracts. Our results of initial fast increase (up to 2 y) followed by modest increase in FA values in CC are in line with a recent DTI study in healthy pediatric subjects. Authors have reported the same time line for FA increase in CC and log linear regression model provided the best fit for WM maturation (29). During the first year of life, infant begins to develop binocular vision and visual accommodation to identify objects. These skills require interhemispheric connections that in turn reflect the maturation of $\mathrm{CC}$ during the first year of life. In the quantitative DTT study of Lebel et al. (13), healthy controls (childhood to adulthood) have shown the earliest and most rapid changes of FA with age in the CC (crucial for left-right hemispheric connections) (30). However, our results are in line with previously published study (17). The population that we studied is very precious. There is no study including earlier study describing changes in FA in whole WM tracts in entire pediatric population.

Limbic system structures are involved in many of our emotions and motivations, particularly, those that are related to survival. Such emotions include fear, anger, and emotions related to sexual behavior. Although CNG is the most prominent WM fiber tract of the limbic system, there have been a very few DTI studies so far describing the maturational changes in CNG. The fornix, involved in very basic processing such as memory and emotion, showed sharp initial increase of FA up to $2 \mathrm{y}$ of life. Yakovlev and Lecours (1) defined the development of myelin in various WM tracts of the human nervous system by using Loyez method for staining myelin and reported that in Fx and CNG, majority of myelination completes during the first year of life.

The descending motor pathway is known to be located in the middle third of the cerebral peduncle and the posterior limb of the internal capsule. The somatosensory radiation is known to pass through the internal capsule posterior to the motor tract. DTI anisotropy maps cannot easily differentiate the motor tract from sensory radiation as the motor and somatosensory tracts are within a band of inferior-superior oriented pathways containing functionally unrelated tracts (31).

The myelination of the sensorimotor tract does not begin until near term age (2). Using Loyez method for staining myelin, Yakovlev and Lecours (1) reported that the majority of myelination in pyramidal tracts completes during the first $2 \mathrm{y}$ of life. Myelination is known to begin earlier in the commissural and projection fibers than in the association fibers, and earlier in the occipital and temporal lobes than in the frontal lobe $(1,32)$. SLF and ILF are among the major long association fibers. SLF is located at the supero-lateral side of the putamen and forms an arc sending branches to the frontal, 
parietal, occipital, and temporal lobes, and ILF connects the extrastriate occipital cortex with lateral temporal cortex, parahippocampal gyrus, and amygdale. In our study, SLF and ILF lagged behind $\mathrm{CC}$, sensory-motor pathways and limbic tracts, and an initial sharp increase in FA was observed up to the third year of life. It is known that ability to solve problems through activities that involve perception, attitude, tone, and movements before the appearance of verbal language develop first during childhood. Subsequently, the child learns to integrate visuospatial information that is associated with SLF. Based on segmentation from T1-weighted images of pediatric and adolescent populations (4-17 y old), Paus et al. (33) reported that the SLF is one of the slowest maturing WM tracts among the major WM tracts of cerebrum.

In addition, we observed significantly high FA values in left CNG compared with their contralateral fiber bundles at all ages that suggest cerebral laterality. A marked left-greater-than-right asymmetry pattern in FA values was observed in the CNG bundles. Anterior $\mathrm{CNG}$ is related mainly to the anterior cingulate cortex, which is associated with neural implementation of attentional control and other functions (34). Anterior cingulate cortex has been studied in details and asymmetric disturbances have been reported in schizophrenic patients (35). The asymmetry of CNG observed in our study is in line with previous fiber tractsbased DTI studies (36) and may contribute to some functional difference between anterior cingulate cortices.

In our study, an increasing trend of FD values was observed in all fiber bundles; however, no regression model provided a significant $R^{2}$ value to determine the changes in FD values with age. Fiber bundle maturation comprises of peripheral axonal sprouting, axonal growth, and on-going myelination. FD, an indicator of fiber bundle thickness at each voxel in human brain, is calculated by dividing the number of fiber paths in the cube by the volume of the cube. On other hand, FA values reflects overall growth of axonal bundle and on-going myelination in addition to fiber bundle thickness that suggest FA is probably a better indicator of fiber maturation compared with FD.

In conclusion, this study demonstrates the normal development of the whole WM fiber bundles and cerebral lateralization in entire pediatric age range by using quantitative DTT that may greatly improve our understanding of the human brain development.

Acknowledgments. Sona Saksena acknowledges the financial assistance from the Indian Council of Medical Research, New Delhi, India.

\section{REFERENCES}

1. Yakovlev PI, Lecours AR 1967 The myelogenetic cycles of regional maturation of the brain. In: Minkowski A (ed) Regional Development of the Brain in Early Life. Blackwell Scientific, Oxford, pp 3-70

2. Barkovich AJ 2000 Concepts of myelin and myelination in neuroradiology. Am J Neuroradiol 21:1099-1109

3. Paus T, Collins DL, Evans AC, Leonard G, Pike B, Zijdenbos A 2001 Maturation of white matter in the human brain: a review of magnetic resonance studies. Brain Res Bull 54:255-266

4. Benes FM 1989 Myelination of cortical-hippocampal relays during late adolescence. Schizophr Bull 15:585-593

5. Morriss MC, Zimmerman RA, Bilaniuk LT, Hunter JV, Haselgrove JC 1999 Changes in brain water diffusion during childhood. Neuroradiology 41:929-934
6. Engelbrecht V, Rassek M, Preiss S, Wald C, Mödder U 1998 Age-dependent changes in magnetization transfer contrast of white matter in the pediatric brain. Am J Neuroradiol 19:1923-1929

7. Le Bihan D, Mangin JF, Poupon C, Clark CA, Pappata S, Molko N, Chabriat H 2001 Diffusion tensor imaging: concepts and applications. J Magn Reson Imaging 13:534-546

8. Beaulieu C 2002 The basis of anisotropic water diffusion in the nervous system-a technical review. NMR Biomed 15:435-455

9. Wieshmann UC, Clark CA, Symms MR, Franconi F, Barker GJ, Shorvon SD 1999 Reduced anisotropy of water diffusion in structural cerebral abnormalities demonstrated with diffusion tensor imaging. Magn Reson Imaging 17:1269-1274

10. Moseley M 2002 Diffusion tensor imaging and aging-a review. NMR Biomed 15:553-560

11. Mukherjee P, Chung SW, Berman JI, Hess CP, Henry RG 2008 Diffusion tensor MR imaging and fiber tractography: technical considerations. Am J Neuroradiol 29:843852

12. Partridge SC, Mukherjee P, Berman JI, Henry RG, Miller SP, Lu Y, Glenn OA, Ferriero DM, Barkovich AJ, Vigneron DB 2005 Tractography-based quantitation of diffusion tensor imaging parameters in white matter tracts of preterm newborns. J Magn Reson Imaging 22:467-474

13. Lebel C, Walker L, Leemans A, Phillips L, Beaulieu C 2008 Microstructural maturation of the human brain from childhood to adulthood. Neuroimage 40:10441055

14. Eluvathingal TJ, Hasan KM, Kramer L, Fletcher JM, Ewing-Cobbs L 2007 Quantitative diffusion tensor tractography of association and projection fibers in normally developing children and adolescents. Cereb Cortex 17:2760-2768

15. Hasan KM, Iftikhar A, Kamali A, Kramer LA, Ashtari M, Cirino PT, Papanicolaou AC, Fletcher JM, Ewing-Cobbs L 2009 Development and aging of the healthy human brain uncinate fasciculus across the lifespan using diffusion tensor tractography. Brain Res 1276:67-76

16. Giorgio A, Watkins KE, Douaud G, James AC, James S, De Stefano N, Matthews PM, Smith SM, Johansen-Berg H 2008 Changes in white matter microstructure during adolescence. Neuroimage 39:52-61

17. Basser PJ, Pajevic S, Pierpaoli C, Duda J, Aldroubi A 2000 In vivo fiber tractography using DT-MRI data. Magn Reson Med 44:625-632

18. Johansen-Berg H, Behrens TE 2006 Just pretty pictures? What diffusion tractography can add in clinical neuroscience. Curr Opin Neurol 19:379-385

19. Catani M 2006 Diffusion tensor magnetic resonance imaging tractography in cognitive disorders. Curr Opin Neurol 19:599-606

20. Oldfield RC 1971 The assessment and analysis of handedness: the Edinburgh inventory. Neuropsychologia 9:97-113

21. Tan U, Tan M 1999 Incidences of asymmetries for the palmar grasp reflex in neonates and hand preference in adults. Neuroreport 10:3253-3256

22. Hasan KM, Parker DL, Alexander AL 2001 Comparison of gradient encoding schemes for diffusion-tensor MRI. J Magn Reson Imaging 13:769-780

23. Mori S, Crain BJ, Chacko VP, van Zijl PC 1999 Three-dimensional tracking of axonal projections in the brain by magnetic resonance imaging. Ann Neurol 45:265-269

24. McGraw P, Liang L, Provenzale JM 2002 Evaluation of normal age-related changes in anisotropy during infancy and childhood as shown by diffusion tensor imaging. Am J Roentgenol 179:1515-1522

25. Schneider JF, Il'yasov KA, Hennig J, Martin E 2004 Fast quantitative diffusiontensor imaging of cerebral white matter from the neonatal period to adolescence. Neuroradiology 46:258-266

26. Jones DK, Symms MR, Cercignani M, Howard RJ 2005 The effect of filter size on VBM analyses of DT-MRI data. Neuroimage 26:546-554

27. Smith SM, Jenkinson M, Johansen-Berg H, Rueckert D, Nichols TE, Mackay CE, Watkins KE, Ciccarelli O, Cader MZ, Matthews PM, Behrens TE 2006 Tract-based spatial statistics: voxelwise analysis of multi-subject diffusion data. Neuroimage 31:1487-1505

28. Wakana S, Caprihan A, Panzenboeck MM, Fallon JH, Perry M, Gollub RL, Hua K, Zhang J, Jiang H, Dubey P, Blitz A, van Zijl P, Mori S 2007 Reproducibility of quantitative tractography methods applied to cerebral white matter. Neuroimage 36:630-644

29. Saksena S, Husain N, Malik GK, Trivedi R, Sarma M, Rathore RS, Pandey CM, Gupta RK 2008 Comparative Evaluation of the cerebral and cerebellar white matter development in pediatric age group using quantitative diffusion tensor imaging. Cerebellum 7:392-400

30. Catani M, Jones DK, Donato R, Ffytche DH 2003 Occipito-temporal connections in the human brain. Brain 126:2093-2107

31. Pajevic S, Pierpaoli C 1999 Color schemes to represent the orientation of anisotropic tissues from diffusion tensor data: application to white matter fiber tract mapping in the human brain. Magn Reson Med 42:526-540

32. Brody BA, Kinney HC, Kloman AS, Gilles FH 1987 Sequence of central nervous system myelination in human infancy: I. An autopsy study of myelination. J Neuropathol Exp Neurol 46:283-301

33. Paus T, Zijdenbos A, Worsley K, Collins DL, Blumenthal J, Giedd JN, Rapoport JL, Evans AC 1999 Structural maturation of neural pathways in children and adolescents: in vivo study. Science 283:1908-1911

34. van Veen V, Cohen JD, Botvinick MM, Stenger VA, Carter CS 2001 Anterior cingulate cortex, conflict monitoring, and levels of processing. Neuroimage 14:1302-1308

35. Benes FM 2000 Emerging principles of altered neural circuitry in schizophrenia. Brain Res Brain Res Rev 31:251-269

36. Gong G, Jiang T, Zhu C, Zang Y, Wang F, Xie S, Xiao J, Guo X 2005 Asymmetry analysis of cingulum based on scale-invariant parameterization by diffusion tensor imaging. Hum Brain Mapp 24:92-98 